\title{
Atrial natriuretic peptide down-regulates neutrophil recruitment on inflamed endothelium by reducing cell deformability and resistance to detachment force
}

Vasilios A. Morikis a , Chris Radecke a, Yanyan Jiang a , Volkmar Heinrich ${ }^{\text {a }}$, Fitz-Roy Curry ${ }^{\mathrm{a}, \mathrm{b}}$ and Scott I. Simon ${ }^{\mathrm{a}}$

${ }^{a}$ Department of Biomedical Engineering, University of California, Davis, CA 95616, USA

${ }^{\mathrm{b}}$ Department of Physiology and Membrane Biology, School of Medicine, University of California, Davis, CA 95616, USA

[Biorheology 52(5,6) 2015, 447-463]

Link to the original article: http://content.iospress.com/articles/biorheology/bir15067.

DOI: 10.3233/BIR-15067

After publication of this article, we noticed that the Acknowledgement Statement in this article was not included. The statement is provided below:

\section{Acknowledgement Statement}

Supported by NIH grants HL28607-32 to FEC and NIH grant AI047294 to SIS. 